\title{
The Isolation and Identification of Mycoplasmas Which cause Contagious Agalactia from Sheep and Goat in Tehran Province
}

\author{
Professor Dr. Sorush Niknamian \\ Board Member of Weston A Price Foundation, Washington Dc, USA \\ E-mail: so.niknamian@gmail.com
}

\begin{abstract}
The Agalactia, has been known as a contagious disease in Mediterranean countries, Africa and Europe, and west Asia e.g. Iran where the livestock were reared massively for approximately several years ago and it has been led to noticeable economic damages and losses. This disease appears by symptoms of fever, inappetence, lameness and loss of milk in dairy ewes, from reduction to total lack of lactation and abortion in pregnant livestock. No study has been so far conducted on isolation of Agalactia disease factor and determining its molecular identity in ovine and caprine herds in Tehran province. The current research aimed to isolate and determine molecular identity of Agalactia disease factor (Agalactiae mycoplasma) in sheep and goat that suffered from Agalactia using culture and PCR methods in Tehran province. Among total 33 taken samples, 14 samples were confirmed as mycoplasma species that showed specific band (bp163) on agarose gel and in one of these 14 given samples, the culture was positive.
\end{abstract}

Keywords: Mycoplasma, Agalactia, Sheep and goat, Tehran province

\section{Introduction}

As a contagious disease, Agalactia, is highly prevalent and one of the infectious diseases of livestock throughout the world and it is always led to exertion of economic losses to livestock populations of sheep and goats. This disease is prevalent among tame livestock and wild animals in many middle eastern and Mediterranean countries (8).

The given disease causes abortion, paralysis and lack of suitable mobility, arthritis, lameness, keratoconjunctivitis and finally slimness and weakness of livestock (7). Although there are not a lot of mortalities caused by this disease, due to long period of morbidity in livestock, it is followed by a lot of economic losses.

Milk production is also never returned to the normal quantity even after curing of livestock. Similarly, high amount of abortion in the cattle is known as one of the economic losses.

The sheep and goat populations are incubated by domestically produced vaccine of Razi Serum and Vaccine Production Institute every year to fight against the aforesaid diseases, but despite regular incubation, some of livestock are still involved in this disease. 
Whereas no study has been yet carried out on isolation and identifying of Agalactia disease factors in Tehran province, the current study is assumed as the first investigation in Agalactia disease among sheep and goats in Tehran province.

Review on results of this study suggests that rate of prevalence of disease has reached to $100 \%$ level among the herd and this indicates spreading disease.

It was characterized by analysis of sensitivity of sheep and goat to mycoplasma that the morbidity rates were $28.6 \%$ and $46 \%$ in goats and sheep, respectively.

Due to geographic situation of Tehran province (livestock traffic intersection) and because of the highest rate of meat consumption in Iran, Tehran province is considered as one of national animal husbandry poles, especially in meat production ranking in Iran.

Despite the aforesaid conditions, there are various livestock diseases in this province including contagious Agalactia (diluted milk). Primarily, one can employ clinical symptoms (incidence of mastitis, arthritis and keratoconjunctivitis) thereby it is possible to determine final diagnosis of disease by isolation of mycoplasma from milk, synovial fluid, tear, ear secretions or eye impaired tissues and then identifying Agalactiae mycoplasma. The mycoplasma infections are generally diagnosed according to serumal tests including biochemical and immunflorescent experiments and or observation of antibody in milk or serum using complementary stabilization elements and or ELISA and or isolation of organism by culture in vitro (10). Anyway, due to creation of intra-species cross- reactions the serumal techniques are interrupted while culture method is time-consuming and it is difficult for isolation of many hard-growing mycoplasmas. The various species can be distinguished using DNA specific tracking of the species, though this technique has improved speed and property of diagnostic methods, it is less sensitive and it can only isolate up to 104 organisms (15).

The germ should be isolated from blood circulation, milk, eye secretions and or joint secretions for final diagnosis. The presence of vectors can be found by milk culture. As usual, milk or suspicious sample is cultured in the specific PPLO broth as basic medium in vitro to which normal horse serum (20\%) and Thallium acetate (1:4000) were added and the sample was stored in the oven at $37^{\circ} \mathrm{C}$ for 3-4 days. Then a drop of this sample was put on surface of PPLO agar as specific solid culture medium in which the usual techniques were used for diagnosis of mycoplasmas mainly based on classic methods e.g. biochemical and immunofluorescent techniques. These techniques are time-consuming and it is often difficult to interpret the results of these tests. At present, there is no standard and reference test thereby the given results can be compared with each other within different experiments.

The researchers showed that the maximum numbers of positive samples $(85 \%)$ were isolated from eye swabs taken from morbid sheep and goat (15). De La Fe et al. isolated Agalactiae mycoplasma from sperms of male goats under healthy conditions using culture and PCR 
techniques for the first time (13). Amores et al. indicated that PCR method was the fast and sensitive technique in diagnosis and isolation of Agalactiae mycoplasma from ear swab of goat (6).

Ghader Sohi et al. conducted a research project titled identification of Agalactiae mycoplasma and other Agalactia creation agents in sheep and goat using PCR and culture in Iran (2006) and in Razi Serum and Vaccine Production Institute to achieve mycoplasma agents for Agalactia disease in sheep and goat. About 2000 samples were tested by PCR experiment and under culture in this project. This study has been carried out in 17 provinces of Iran. The aforesaid samples were taken from healthy and morbid animals and the milk, eye swab and joint synovial fluid were utilized as samples (4). In another investigation that was conducted by Bidhendi et al., isolation and identification of Agalactiae mycoplasma taken from milk of sheep and goats in Kurdistan province were mentioned as the objective of this study. Finally, 5 isolates were used for determining nucleotide sequence out of which one of them (HQ722028 was recorded in gene bank (5).

Table 1: Sheep and goat mycoplasmas (3)

\begin{tabular}{|c|c|c|c|}
\hline Host & Intensity & Morbidity (if created) & Species \\
\hline Sheep and goat & High & Arthritis, conjunctivitis, mastitis & M. agalactiae \\
\hline Multiple hosts & Low & Mucus & M. arginini \\
\hline Sheep and goat & High & $\begin{array}{l}\text { Mastitis, arthritis, septicemia in } \\
\text { young goat }\end{array}$ & M. capricolum \\
\hline Sheep and goat and ibex & Medium & Keratoconjunctivitis & M. conjunctivae \\
\hline Goat & High & Pleurisy and pneumonia & $\begin{array}{l}\text { M. Mycoides S.G. } \\
\text { Caprie }\end{array}$ \\
\hline Sheep and goat & High & $\begin{array}{l}\text { Septicemia, arthritis, mastitis, and } \\
\text { conjunctivitis }\end{array}$ & $\begin{array}{l}\text { M. mycoides S.G. } \\
\text { Mycoids }\end{array}$ \\
\hline Sheep and goat & Low & $\begin{array}{l}\text { Chronic pneumonia by the aid of } \\
\text { pasteurella }\end{array}$ & M. ovipneumoniae \\
\hline Goat & Medium & Mastitis & M. putrefaciens \\
\hline Goat & High & $\begin{array}{l}\text { Septicemia, pleurisy and } \\
\text { pneumonia }\end{array}$ & M. strain F38 \\
\hline
\end{tabular}

\section{Materials and methods}

The processes of execution of this study were as follows:

The studied population in this study included all morbid sheep and goats (suspicious to Agalactia) in Tehran province and purposive sampling was conducted. As a result, the suspicious herds were sampled in Tehran province during four different seasons for two years and after isolation of agent, kind and species of agent bacterium were confirmed and the molecular identity of isolated strains were determined.

It was initially talked to and consulted with the related officials (heads of departments for analysis and fighting with livestock diseases in veterinary networks)in Tehran Province 
Veterinary Administration in this study and about method of identification and diagnosis of suspicious herds to disease, collection of statistical data and sampling technique and transferring them into media with content of stored substances (PPLO broth). Then a briefing session was held for veterinarians in medical units by analysis and fighting against livestock diseases in private and public sectors and to train them the way of reporting morbidity of diseases. Following to report of morbidity focus, the responsible colleagues visited the patient herds and completed the appended questionnaire if they confirmed clinically their morbidity. Those studied herds, which were studied, were not vaccinated against Agalactia at least during the last year. Depending on the involved area, the aseptic morbidity samples were collected from milk and eye swab, ear swab, secretion of aborted fetus and or pleural secretions of ill livestock and transferred to transport medium including medium (red phenol $0.2 \%+$ glucose $0.1 \%+$ ampicillin $200 \mathrm{IU} / \mathrm{ml}+$ yeast extract $10 \%+$ serum of heart infusion broth $+20 \%$ ) and immediately sent to Razi Serum and Vaccine Production Institute under cold PCR conditions for isolation and identification of strain.

Two culture techniques (in fluid medium and on agar culture medium) have been utilized in parallel in this study for isolation of disease agent for diagnosis of positive cases out of which totally 33 morbid cases were extracted from 33 ill sheep and goats.

\section{Results}

The results of each of tests have been separately recorded and 14 cases were positive in PCR test. As it observed in Table 2, the samples were extracted from 5 suspicious herds.

Table 2: Frequency of herds based on results of culture, PCR and type of mycoplasma

\begin{tabular}{ccc}
\hline & Culture & PCR for mycoplasma strain \\
\hline+ & 1 & 5 \\
- & 4 & 0 \\
Total & 5 & 5 \\
\hline
\end{tabular}

At least one out of total 4 herds in this study was isolated as sample with Agalactiae mycoplasma and no positive case was found in a herd. In other words, the herd infection rate by Agalactiae mycoplasma is $20 \%$. As it seen in Tables 3 and 6, 33 patient sheep and goats were sampled and mycoplasma species was isolated from 14 cases in the given test.

Among 26 sampled suspicious sheep to disease, mycoplasma strain was isolated from 12 cases in PCR test. In other words, the rate of infection with mycoplasma strain is $46.15 \%$ in the sampled sheep. Among total 7 sampled ill goats, mycoplasma strain was isolated from 2 cases in PCR test. Namely, the rate of contamination to mycoplasma strain is $28.57 \%$ in the sampled goats. 
Table 3: Frequency of PCR test results of mycoplasma strain separately for sheep and goat

\begin{tabular}{ccc}
\hline & Number of sampled livestock & PCR for mycoplasma strain \\
\hline Sheep & 26 & 12 \\
Goat & 7 & 2 \\
Total & 33 & 14 \\
\hline
\end{tabular}

Totally 33 morbid samples were taken in this study and sent to mycoplasma reference laboratory in Razi Serum and Vaccine Production Institute. The extracted samples included eye swab, milk (Table 5). The analysis on results of the extracted samples (Table 4) indicates that 19 cases were negative in culture and PCR test. In other words, despite clinical symptoms, $57.57 \%$ of cases were negative. Likewise, 13 samples were positive in PCR test and 1 case was positive both in culture and PCR test.

As it seen in Table 5, out of total 33 extracted morbid samples, the highest frequency belongs to the milk (66.5\% of case) and the lowest frequency is related to eye swab.

Table 4: Microscopic image (40x) of observed colonies on PPLO Agar medium

\begin{tabular}{ccc}
\hline Culture & PCR & Number of sample \\
\hline+ & + & 1 \\
- & - & 19 \\
+ & - & 0 \\
- & + & 13 \\
Total & & 33 \\
\hline
\end{tabular}

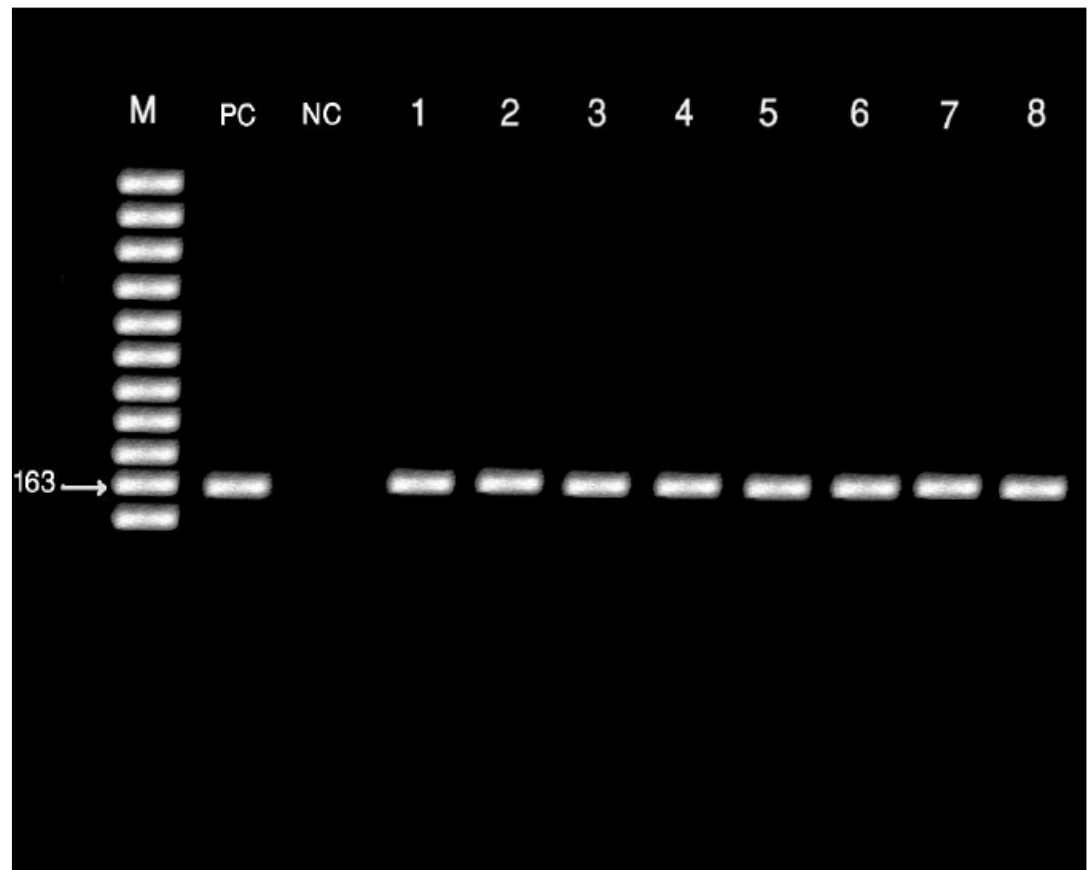

Figure 1: Image of electrophoresis gel of PCR product using specific promoter of Agalactiae mycoplasma species (FS1_FS2) on bp375 strains may be observed in 8 samples of positive case. 
The highest frequency of positive cases belongs to eye $(45.45 \%)$ and the lowest frequency of them is related to the milk (40.9\%) on sampling locations among 14 cases of positive mycoplasma species in PCR test.

It was characterized in this study that despite morbidity of livestock in $42 \%$ of the morbid cases, mycoplasma species was not isolated according to the symptoms attributed to Agalactia in the culture and PCR test and this indicated necessity for paying attention to other infectious factors that might create symptoms similar to Agalactia.

Table 5: The rate of frequency for isolation of mycoplasma species from milk and eye

\begin{tabular}{ccccc}
\hline Quantity & $\begin{array}{c}\text { PCR for mycoplasma } \\
\text { strain }\end{array}$ & $\begin{array}{c}\text { PCR - Agalactia } \\
\text { species- } \\
\text { frequency of } \\
\text { positive type }\end{array}$ & $\begin{array}{c}\text { PCR - Agalactia } \\
\text { species }\end{array}$ \\
\hline Eye swab & 11 & 5 & 45.45 & 3 \\
Milk & 22 & 9 & 40.9 & 1 \\
\hline
\end{tabular}

\section{Discussion and conclusion}

The Agalactia disease is highly prevalent in the world as one of the infectious diseases and is always led to economic losses in livestock populations, especially sheep and goat.

The review on background of disease in Iran and Tehran province confirms this point that the given disease causes a lot of economic losses and damages for livestock population in Iran and Tehran province every year.

The sheep and goat populations are incubated by domestically produced vaccine from Razi Serum and Vaccine Production Institute to fight against the given disease every year but despite conducting regular incubation in some of herds, they are still involved in disease.

Whereas no study has been yet carried out on isolation and identification of agents of Agalactia in Tehran province, the present research is assumed as the first study on Agalactia disease in sheep and goat in Tehran province.

The analysis on results of this study indicates that prevalence of disease in herd may be $100 \%$ and this shows problem of spreading disease.

Only the milk has been used for isolation of the aforesaid factor in the similar studies done in Iran and neighbor countries (5). Due to high sensitivity, specificity of method, fast diagnosis and ease of study and analysis, all these factors have caused the researchers to tend to molecular studies in using PCR technique during recent years.

Among others, Tola et al. (16) isolated and identified Agalactiae mycoplasma using PCR technique on milk samples from the patients with mastitis in four areas in Italy and concluded 
that this method was a fast and specific technique for isolation of Agalactiae mycoplasma. In similar investigations done in Jordan and Morocco, the researchers could also isolate species of Agalactiae mycoplasma from the ill sheep and goats by means of PCR technique. Many efforts have been also made in Iran for isolation and identification of mycoplasma agents of Agalactia using PCR technique, including Pirali and Ibrahami sampled tear and milk from 26 herds and used PCR technique for analysis and isolated 20\% of Agalactiae mycoplasma bacteria (14). Using culture and PCR technique, Moradi et al. could isolate 12 cases of mycoplasma out of 367 milk samples taken in Kurdistan province among them 5 cases were Agalactiae (5).

By employing culture and PCR technique, Kheirkhah et al. managed to isolate 8 cases of mycoplasma out of 17 milk samples taken in Baft city out of which one was Agalactiae species (2).

According to the following reasons, culture and PCR techniques have been utilized to search for mycoplasma factor of diseases in this study:

1) It is recommended to use immunological methods to search for mycoplasma compared to other technique because it needs to increase antibody carat in serumal methods where they emerge 10-40 days after starting clinical symptoms. Furthermore, one may not also search for mycoplasma during incubation period. The serumal cross-reactions are assumed as a serious problem as well and it influences in sensitivity of this reaction further (13).

2) Firstly, adaption of culture methods solely needs for two weeks or more for incubation. Secondly, it necessitates for conventional staining methods (Dienes etc.) and laboratory tests (biochemical ...) for diagnosis of mycoplasma species which lack high precision and sensitivity and there is high possibility for error in such tests.

As one of advanced techniques in diagnosis of microbial factors, PCR reaction was employed in this study.

This study showed that the best location for sampling and isolation of disease factor (Agalactiae mycoplasma) are mastoid and ocular secretions. It was announced in studies of various researchers that Agalactiae mycoplasma was the main factor for Agalactia disease (12).

However similar to the findings of Ghader Sodi et al. and Kheirkhah et al., The other mycoplasmas were the main agent for Agalactia in this study and only $21 \%$ of isolated mycoplasma species included Agalactiae species. The given finding confirms the slight effect of current vaccine in Tehran province and other provinces in Iran including Qom and Kerman provinces because mycoplasma species in this vaccine is not totally consistent with circulating species. 
It has been identified by analysis on rate of sensitivity of sheep and goats to Agalactiae mycoplasma that the rate of morbidity in goats (36\%) was greater than in sheep (14.94\%).

It was characterized in this study that despite illness of livestock with attributed symptoms to Agalactia in $36 \%$ of the extracted morbid cases but mycoplasma was not isolated in culture and PCR where this point emphasizes in necessity for paying attention to other infectious factors that can create symptoms similar to Agalactia. Among the reasons for lack of bacterial growth in culture medium, one can refer to non-observance of the appropriate conditions for extraction, storage and sending sample, absence of mycoplasma in the taken sample due to involvement in other pathogens with similar symptoms, use of corton and or antibiotic by animal-farmer before sampling and small quantity of the grown mycoplasmas in culture medium that might not have been examined and also the slow and hard growth trend for mycoplasmas.

Similar to surveys of Tola et al. (16), the present study showed that highest quantity of isolation of Agalactiae species belonged to the milk samples and synovial fluid but the maximum rate of isolation of mycoplasma species was related to nose secretions, supramastoid lymphatic glands and pleural tissue.

The milk was only used for isolation of given factor in similar studies conducted in Iran and neighbor countries (5). Whereas 79\% of isolated mycoplasmas were non-Agalactiae for this reason the complementary studies are under execution process to identify them.

This study showed that the best sampling location for isolation of disease agent (Agalactiae mycoplasma) were mastoid secretions and synovial fluids respectively.

In order to complete the conduct studies, it is necessary to carry out studies for identifying and determining molecular identity of isolated mycoplasma factors and molecular analysis on isolated Agalactiae strains and determining rate of similarity to the existing strains in vaccine.

\section{Acknowledgement}

The authors appreciate the efforts and measures made by the respected officials in agricultural researches center and veterinary administration of Tehran province and announce there is no conflict of interests in this project.

\section{References}

[1] Hassani Tabatabaei, A. Firouzi, R (2001). 'Bacterial diseases of livestock', Publication and printing institute of University of Tehran, $1^{\text {st }}$ Ed, pp. 469-484.

[2] Kheirkhah, B, Pourbakhsh, SA, Amini, K (2011). 'Isolation of Agalactiae mycoplasma in sheep with Agalactia in Baft city', Journal of comparative pathobiology, accepted paper. 
[3] Shimi, A (1997). 'Veterinary bacteriology and bacterial diseases', Academic Jihad Pub, $1^{\text {st }}$ Ed, pp. 446-456.

[4] Ghader Sohi, A, Akhlaghi A, Fayazi Z, Naserirad AA, Vand Yousefi J (2006). 'Identification of Agalactiae mycoplasma and other Agalactia pathogens in sheep and goat by PCR and culture in Iran', Journal of Razi Serum and Vaccine Production Institute, pp. 10-12.

[5] Moradi Bidhendi S, Khaki P, Pilehchian Langeroodi, R (2010). 'Isolation and identification of Agalactiae mycoplasma using culture and PCR techniques from milk samples of sheep and goat in Kurdistan province', Archive of Razi Institute, 66, pp. 11-16.

[6] Amores J, Corrales JC, Martin AG (2009). Comparison of culture and PCR to detect Mycoplasma agalactiae and Mycoplasma mycoides subsp. Capri in ear swabs taken from goats. Vet. Mic. 102: $42-48$.

[7] Cokrevski S, Crcev D, Loria GR, Nicholas RAJ (2001). Outbreaks of contagious agalactia in small ruminant in the republic of Macdonia. Vet. Rec. 148: 667-670.

[8]Contreras AC, Luengo A, Corrales JC ( 2003). The role of intramamary pathogens in dairy goats, Livestock Pro. Sci. 9: 273-283.

[9] Corrales J, Ensal A, De La Fe C, Sanchez A, Assuncao P, Poveda J, Contreras A (2007). Contagious agalactia in small ruminants. Small Ruminant Res. 68:154-166

[10] Cottew GS (1979). Caprine-ovine mycoplasmas. In: Tully JG, Whitcomb RF. the mycoplasmas. Human and animal mycoplasmas, ed., Academic press pp: 103-132.

[11] DaMassa AJ, Brooks DL, Holmberg CA (1984). Pathogenicity of Mycoplasma capricolum and Mycoplasma putrefaciens. Isr. J. Med. Sci. 20: 975-978.

[12] Dedieu L, Mady V, Lefevre PC (1995). Development of two PCR assays for the identification of mycoplasmas causing contagious agalactia. FEMS Microbiol. Lett. 129: 243-249.

[13] De La Fe C, Assuncao P, Rosales RS, Antunes T, Poveda JB (2006). Characterisation of Protein and antigen variability among Mycoplasma Mycoides subsp Mycoides LC and Mycoplasma agalactiae field strains by SDS-PAGE and immunoblotting. Vet. J. 171: 532-538.

[14] Pirali, K., Ebrahimi,A. 2007. Investigation ofMycoplasma agalactiae in milk and conjunctival swab samples (In Persian) from Sheep !ocks in west central, Iran. Pakistani Journal of Biological Sciences, 10(8), 1346-1348.

[15] Gobel UB, Geiser A, Stanbridge FJ (1987). Oligonucleotide probes complementary to two variable regions of ribosomal RNA discriminate between Mycoplasma species. J. Gen. Microbiol. 133: 1969-1974.

[16] Tola S, Angioi A, Rocchigiani AM, Idini G, Manunta D, Galleri G, Leori G (1997). Detection of Mycoplasma agalactiae in sheep milk samples by Polymerase Chain Reaction. Vet. Mic. 54(1): 17-22.

[17] Tola S, Manunta D, Rocca S, Rocchigiani AM, Idini G, Angioi A, Leori G (1999). Experimental vaccination against Mycoplasma agalactiae using different inactived vaccines. Vac. 17: 2764-2768. 
[18] Zendulkova D, Madanat A, Lany P, Pospisil Z, Ball HJ (2004). Detection of Mycoplasma agalactiae antigen in sheep and goats by monocolonal antibody-based sandwich ELISA, Acta. Vet. Brono. 73: 461-464. 